\title{
Platelet Rich Plasma Intra Articular Injection for Chronic Synovitis Treatment in Patients with Haemophilia One Year Follow Up
}

\author{
María Eulalia Landro*1, Carla Daffunchio ${ }^{1,2}$, Guillermo Cambiaggi ${ }^{1,2}$, Gustavo Galatro ${ }^{1,2}$, Pablo Salgado ${ }^{1}$, Egle \\ Honorat $^{2}$ and Horacio Cavigliaa ${ }^{1,2}$
}

${ }^{1}$ Department of Orthopaedics and Traumatology, Dr. Juan A. Fernández General Hospital, CABA-Argentina

${ }^{2}$ Argentinian Haemophilia Foundation, CABA Argentina

Received: 眥: December 15, 2018; Published: 制: January 09, 2019

*Corresponding author: María Eulalia Landro, Department of Orthopaedic and Traumatology, Dr. Juan A. Fernández General Hospital, CABA-Argentina

Keywords: Synovitis; Platelet Rich Plasma; Haemophilia; Growth Factor; Joint

Abbreviations: PWH: Patients with Haemophilia; IL: Interleukin; TNF: Tumor Necrosis Factor; PRP: Platelet Rich Plasma; PDGF: Platelet Derived Growth Factor; FGF-2: Fibroblast Growth Factor 2 VEGF: Vascular Endothelial Growth Factor; VAS: Visual Analogue Scale; ROM: Range of Movement; JP: Joint Perimeter measure; NSAIDs: Non-Steroidal Anti Inflammatory Drugs; SPSS: Statistical Package for the Social Sciences

\section{Introduction}

In patients with haemophilia ( $\mathrm{PWH})$, frequent bleed events (2-3 in a six-month period) at the same joint site are associated with chronic inflammation of the synovial membrane and reduced mobility due to the increased volume of fluid within the joint [1]. Joint bleeding generally occurs when children begin walking (median age 20 months), which suggests that mechanical forces play a role in initiating a bleed [2]. The synovial membrane of PWH appears to adapt to the presence of iron. Chronic synovitis contributes significantly to joint deterioration in the long-term and leads to chronic pain, disability, and arthropathy [3]. This induces synovial changes such as inflammation, hyperplasia, and angiogenesis. If only one bleed occurs these changes can be transient, as the cartilage regenerates if chondrocytes remain vital. Only the vascular changes are irreversible [4]. The pathogenesis of cartilage damage caused by bleeding is not yet fully understood. Blood-derived mononuclear cells produce cytokines such as interleukin (IL) $1 \mathrm{~b}$ and tumor necrosis factor (TNF). These cells activate synoviocytes, stimulate chondrocytes, and induce biological activities such as hyperplasia with intense neovascularization and inflammation of the synovial membrane [4, 5]. This hypervascularization induces a recurring cycle of persistent hyperplasia and inflammation. The cartilage is damaged as a result of contact with blood and of synovial changes, and cartilage matrix components deteriorate due to synovial production of pro-inflammatory cytokines and proteases.
The cartilage is permanently damaged by chondrocyte apoptosis [6]. Chondrocytes produce hydrogen peroxide after phagocytosis of erythrocytes by macrophages. The resulting chondrocyte apoptosis affects the viability of the extracellular matrix [5]. In vitro studies have shown that Interleukin- $1 \beta$ can prevent cartilage degradation and chondrocyte apoptosis [5]. Platelet-rich plasma (PRP) is used to stimulate healing of the joint and provide symptomatic relief $[7,8]$. This treatment relies on the biological properties of PRP, which contains alpha-granules with growth factors and proteins used in joint homeostasis, healing mechanisms and tissue regeneration $[9,10]$. Platelet derived growth factor (PDGF), transforming growth factor $\beta 1$ (TGF $\beta 1$ ), insulin like growth factor (IGF), platelet factor 4 (PF-4), fibroblast growth factor 2 (FGF-2) and vascular endothelial growth factor (VEGF) accelerate the natural healing process and promote cartilage repair [11]. Alpha granules also contribute cytokines, chemokines and proteins involved in chemotaxis, cell proliferation and maturation, and in controlling inflammatory processes [9]. In addition to alpha granules, platelets contain dense granules with ADP, ATP, calcium ions, histamine, serotonin and dopamine which also contribute to tissue regeneration [12]. Platelets also contain lysosomal granules. These granules secrete molecules such as acid hydro-lases, cathepsin D and E, elastases and lysozyme $[12,13]$. 
All the cytokines and growth factors released by PRP favor resident stem cell recruitment to the site of injury. At the site, these cells secrete additional growth factors and anti-inflammatory cytokines. The recruited stem cells can differentiate into cartilage and replace injured cells [14]. Growth factors and proteins have been found to take part in regulating articular cartilage, stimulating cartilage matrix synthesis, and counteracting the effects of catabolic cytokines such as interleukin-1 and tumor necrosis factor- $\alpha$ [15]. Numerous clinical trials indicate that PRP is a promising treatment for cartilage injuries and joint inflammation, but its role in cartilage repair is not yet fully understood [14]. The aim of this study is to show the results of treatment of chronic synovitis in PWH by means of intra-articular PRP injection at one-year follow-up.

\section{Patients and Methods}

Thirty-nine patients from the Foundation of Haemophilia with chronic synovitis in 66 joints were treated and followed for one year at the Juan A. Fernandez General Hospital in Buenos Aires, Argentina. Thirty-eight patients were haemophilia type A and one type B. Thirty-three patients were severe (84.6\%), and 6 were moderate (15.4\%). Four patients with five synovitis joints (3 knees, one ankle and one elbow) had inhibitors. Three patients had factor VIII inhibitors and one had factor IX inhibitor. This complication represents $10.25 \%$ of the study population (Table 1). Mean age was 25 years (8-48). The affected joints were: 38 (57.6\%) knees, 15 (22.7\%) elbows and 13 (19.7\%) ankles. Twenty-one (53.84\%) patients had synovitis in only one joint, 17 (43.6\%) had synovitis in two joints, and one $(2.56 \%)$ patient had 3 joints with synovitis. The study followed the guidelines of the Declaration of Helsinki and was approved by the hospital's ethics committee. All patients provided written informed consent.

Table 1: Study population data.

\begin{tabular}{|c|c|c|c|c|}
\hline Patients & Inh & Age & Haemophilia & Grade \\
\hline 1 & No & 24 & A & M \\
\hline 2 & Yes & 8 & A & S \\
\hline 3 & Yes & 25 & A & M \\
\hline 4 & No & 32 & A & S \\
\hline 5 & No & 48 & A & S \\
\hline 6 & No & 40 & A & S \\
\hline 7 & No & 20 & A & S \\
\hline 8 & Yes & 11 & A & S \\
\hline 9 & No & 24 & A & S \\
\hline 10 & No & 34 & A & M \\
\hline 11 & No & 20 & A & S \\
\hline 12 & No & 13 & A & S \\
\hline 13 & Yes & 14 & B & S \\
\hline 14 & No & 22 & A & S \\
\hline 15 & No & 38 & A & S \\
\hline 16 & No & 20 & A & S \\
\hline 17 & No & 33 & A & M \\
\hline 18 & No & 29 & A & S \\
\hline 19 & No & 30 & A & S \\
\hline & & & & \\
\hline
\end{tabular}

\begin{tabular}{|c|c|c|c|c|}
\hline 20 & No & 8 & A & $\mathrm{S}$ \\
\hline 21 & No & 22 & A & $S$ \\
\hline 22 & No & 9 & $\mathrm{~A}$ & $\mathrm{~S}$ \\
\hline 23 & No & 22 & A & $\mathrm{S}$ \\
\hline 24 & No & 25 & $\mathrm{~A}$ & $\mathrm{~S}$ \\
\hline 25 & No & 32 & A & M \\
\hline 26 & No & 35 & A & $S$ \\
\hline 27 & No & 18 & $\mathrm{~A}$ & $\mathrm{~S}$ \\
\hline 28 & No & 25 & $\mathrm{~A}$ & S \\
\hline 29 & No & 16 & $\mathrm{~A}$ & $\mathrm{~S}$ \\
\hline 30 & No & 27 & $\mathrm{~A}$ & $\mathrm{~S}$ \\
\hline 31 & No & 35 & A & $S$ \\
\hline 32 & No & 20 & $\mathrm{~A}$ & $\mathrm{~S}$ \\
\hline 33 & No & 28 & A & $\mathrm{S}$ \\
\hline 34 & No & 22 & A & $\mathrm{M}$ \\
\hline 35 & No & 28 & $\mathrm{~A}$ & $\mathrm{~S}$ \\
\hline 36 & No & 35 & A & $S$ \\
\hline 37 & No & 33 & $\mathrm{~A}$ & $\mathrm{~S}$ \\
\hline 38 & No & 26 & $\mathrm{~A}$ & $\mathrm{~S}$ \\
\hline 39 & No & 13 & A & $S$ \\
\hline
\end{tabular}

Note: $\mathrm{S}=$ severe, $\mathrm{M}=$ Moderate

\section{Inclusion Criteria}

Patients with chronic synovitis diagnosed by clinical exam, ultrasound and/or MRI.

\section{Exclusion Criteria}

Patients with grade 5 arthropathy (osseous ankylosis) according to Arnold and Hilgartner's radiological classification and clinical exam. Patients with loss of the skin or active infection in the joint were also excluded. The following parameters were evaluated: Visual Analogue Scale (VAS), number of Bleeding Episodes in the last 3 months (BE), Haemophilia Joint Health Score (HJHS) for each affected joint, Range of Movement (ROM) and Joint Perimeter measure (JP), before treatment, 3 months, 6 months and 12 months after treatment. The knee joint perimeter was measured at two points: $\mathrm{K} 1$ in the center of the patella, and $\mathrm{K} 23 \mathrm{~cm}$ above the top edge of the patella. The perimeter of ankle and elbow joints were measured at only one point. Elbows were measured around the medial and lateral epi-condyle and for ankles just distal to the tip of the malleolus [16]. Clinical evaluations were performed by the same interdisciplinary team of haematologists, traumatologists, and physiotherapists each time.

\section{Platelet Rich Plasma Preparation and Application}

Preparation procedures were identical to those in our previous study [17]. In brief, autologous intravenous blood was obtained using a BD vacutainer $₫$ collection set. After blood extraction each patients received their substitutive factor, a bolus dose of FVIII/FIX to bring the factor level to $50 \%$. They continued to receive enough factor to maintain a level of $30 \%$ in the following two days. In patients 
with inhibitors, an initial dose of recombinant FVIIa (rFVIIa) 150 $200 \mu \mathrm{g} / \mathrm{kg}$ immediately before the injection was infused, followed by three doses of $90 \mu \mathrm{g} / \mathrm{kg}$ every 3 hours. Then, a high daily dose $(200-300 \mu \mathrm{g} / \mathrm{kg})$ was given the following three days. The extracted blood was centrifuged for 8 minutes at $1600 \mathrm{rpm}$ (Presvac $\AA$ centrifuge, Bs. As.- Argentina) : Prevac ${ }^{\circledR}$ centrifuge). PRP was separated under sterile conditions and placed in a syringe. One $\mathrm{ml}$ of the concentrate was reserved for counting platelets in a Coulter LH 750 Analyzer (Beckman Coulterष区 Inc, USA). To perform the injection, the joint area was embrocated with iodinepovidone and covered with a fenestrated sterile drope. A mean volume of $4 \mathrm{ml}$ of fresh PRP was injected into the joint cavity. Joint aspiration was done before PRP injection if required. No antibiotic prophylaxis was administrated. Patients can take paracetamol if needed. Nonsteroidal anti-inflammatory drugs (NSAIDs) interfere with platelet segregation and function [18]. It is an outpatient procedure, so patients can continue with their regular activities after treatment.

\section{Statistics}

Statistical analysis of the data was performed using the Statistical Package for the Social Sciences (SPSS). Wilcoxon test for paired samples and the Friedman test with Bonferroni correction were performed. Fisher's exact test was used to evaluate the effect of treatment. It compares the same parameters before and after treatment. ANOVA was also used. A p-value below 0.05 was considered statistically significant.

\section{Results}

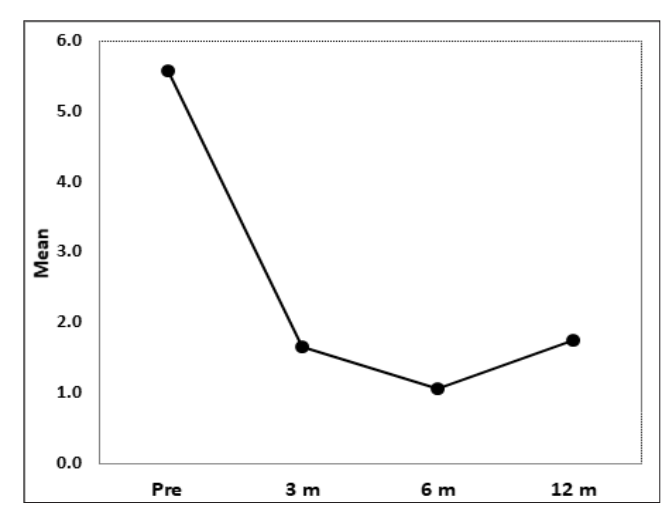

Figure 1: Visual Analogue score. Before treatment the mean VAS was 5.57, 1.64 (SD: 0.21) three months after treatment, 1.05 six months after treatment, and 1.75 twelve months after treatment. The VAS-scores for pain perception were statistically significant $(\mathrm{p}<0.001)$.

An average 15ml (10-35) of blood was extracted for the PRP preparation. An average $4 \mathrm{ml}(2-9 \mathrm{ml})$ of PRP was injected in each joint, depending of the size of the joint. Platelet mean count was $420103 / \mathrm{ml}$ (200-850 platelets/ml), two to three times more than baseline. All patients reported pain relief and decrease in joint bleeding episodes. The VAS-scores for pain perception were statistically significant $(\mathrm{p}<0.001)$. Before treatment the mean VAS was 5.57 (SD: 0.30), 1.64 (SD: 0.21) three months after treatment, 1.05 (SD: 0.22) 6 months after treatment, and 1.75 (SD: 0.40) 12 months after treatment (Figure 1) Decrease of joint bleeding episodes (BE) was also statistically significant $(\mathrm{p}<0.001)$ according to the frequency of joint bleedings episodes before and after treatment. The average number of $\mathrm{BE}$ was 2.47 before treatment, 0.29 after 3 months treatment, 0.35 after 6 months treatment and 0.65 after 12 months treatment (Figure 2). Only three (4.54\%) joints had been free of bleeding episodes before treatment. Fiftyfour $(81.8 \%)$ of joints were free of bleeding episodes three months after treatment, 52 joints ( $78.78 \%$ ) six months after treatment, and $43(65.15 \%)$ one year after treatment.

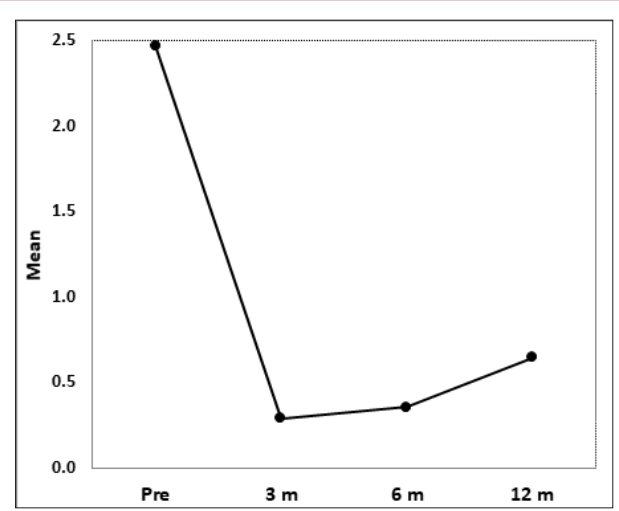

Figure 2: Bleeding Episodes. The average number of $\mathrm{BE}$ was 2.47 before treatment, 0.29 after three months treatment, 0.35 after six months treatment and 0.65 after twelve months treatment. This differences were statistically significant $(\mathrm{p}<0.001)$.

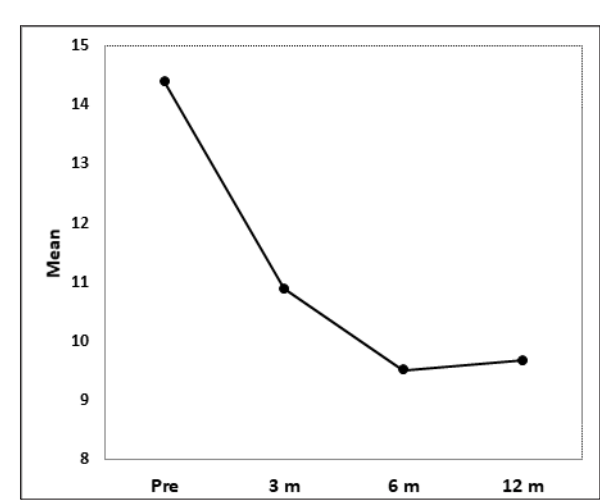

Figure 3: The Haemophilia Joint Health Score (HJHS) average was 14.38 before treatment, 10.88 three months after treatment, 9.51 six months after treatment, and 9.67 twelve months after treatment. The differences in HJHS pre and post treatment were statistically significant $(\mathrm{p}<0.001)$.

The Haemophilia Joint Health Score (HJHS) average was 14.38 (SD: 0.47) before treatment, 10.88 (SD: 0.47) 3 months after treatment, 9.51 (SD: 0.49) 6 months after treatment, and 9.67 (SD: $0.59) 12$ months after treatment. The differences in HJHS pre and post treatment were statistically significant $(\mathrm{p}<0.001)$. (Figure 3) Range of motion improved for all patients but there were no statistically differences. Joint perimeter measures also improved 3 months, 6 months and 12 months after treatment. The difference of the joint perimeter measure before and after treatment was statistically significant for ankle and elbow joints $(\mathrm{p}=0.011, \mathrm{p}=0.008$ respectively). Knees were the most affected joint. The change in the 
K1 perimeter was not showed statistically significant before and after treatment. The change in the K2 perimeter was statistically significant for knee joints (Table 2). Although parameters as BE,
VAS, JP, and HJHS remain below pre-treatment levels, they are slightly higher al twelve months than at 6 months.

Table 2: Joint perimeter measure before and after treatment.

\begin{tabular}{|c|c|c|c|c|c|}
\hline Joint Perimeter & Before & $\mathbf{3 m}$ & $\mathbf{6 m}$ & $\mathbf{1 2 m}$ & $\mathbf{p}$ \\
\hline Knee K1 & $37,6 \pm 3,0$ & $36,2 \pm 3,1$ & $36,1 \pm 3,2$ & $36,3 \pm 3,5$ & NS \\
\hline Knee K2 & $36,7 \pm 4,2$ & $34,9 \pm 4,2$ & $34,7 \pm 4,4$ & $35,0 \pm 4,6$ & 0,001 \\
\hline Elbow & $26,0 \pm 2,8$ & $25,4 \pm 2,4$ & $25,2 \pm 2,4$ & $25,1 \pm 2,4$ & 0,011 \\
\hline Ankle & $25,8 \pm 2,5$ & $25,0 \pm 2,3$ & $24,9 \pm 2,3$ & $25,1 \pm 2,2$ & 0,008 \\
\hline
\end{tabular}

Note: NS: Not significant. K1 (Knee perimeter 1) was not showed statistically significant differences before and after treatment.

Joint perimeter measure was statistically significant for K2 (Knee perimeter 2), ankle and elbow joints.

\section{Discussion}

Platelets contain growth factors which regulate the tissue healing process. This study included: 38 (57.6\%) knee, $15(22.7 \%)$ elbow and 13 (19.7\%) ankle joints. No complications were observed after blood extraction, and no joint bleeding occurred as a result of intraarticular PRP injection. The VAS score showed a statistically significant decrease in pain, throughout the entire study period. The number of BE diminished significantly in the third month, and continued bellow pretreatment levels a year. Only three $(4.54 \%)$ joints had been free of bleeding episodes before treatment. Fiftyfour $(81.8 \%)$ of joints were free of bleeding episodes three months after treatment, 52 joints $(78.78 \%)$ six months after treatment, and $43(65.15 \%)$ one year after treatment. The HJHS was also significantly lower after treatment. Ankle, elbow and K2 joint perimeter decreased significantly before and after treatment. The change in K1 perimeter was not statistically significant before and after treatment. Knee joint perimeter was related to the location of the synovial fluid. The proliferation of synovial tissues occurs primarily in the suprapatellar region so K2 perimeter is more sensitive to changes in the volume of synovial fluid, and the inflammation of synovial membrane. Although parameters as $\mathrm{BE}, \mathrm{VAS}, \mathrm{JP}$ and HJHS remain below pretreatment levels they are slightly higher at twelve months than at six months. Repeating the PRP injection once a year could help to avoid progression of these clinical parameters and protect the joint.

We recommend the use of PRP for synovitis treatment since platelets secrete growth and coagulations factors, adhesion molecules, cytokines, chemokines and integrins. We believe PRP is a useful tool to treat chronic synovitis in PWH. After activation, concentration of growth factors increases significantly. Anabolic cytokines like transforming growth factor- $\beta$, IGF, basic fibroblast growth factor and PDGF promote and protect chondrocytes and stimulate chondrocyte and mesenchymal stem cell (MSC) proliferation. These stem cells can differentiate into chondrocytes and diminish the catabolic effects of inflammatory cytokines, such as IL-1 [19]. Platelets also secrete inflammatory mediators and modulators, and anti-inflammatory cytokines like IL-1, tumor necrosis factor (TNF) receptor (sTNF-R) I and II, IL-4, IL-10, IL13 , and interferon [20]. IL-1ra inhibits the bioactivity of IL-1 by blocking its receptors. TNF-RI and sTNF-RII can bind to free TNF $\alpha$ preventing signal transduction [21]. IL-4, IL-10, and IL-13 can increase IL-1ra production and reduce TNF $\alpha$ [22]. PRP also releases pro-inflammatory cytokines such as IL- $1 \alpha$, IL-1 $\beta$, TNF $\alpha$, IL-6, IL-8, IL-17, and IL-18, but their concentrations are much lower than those of anti-inflammatory cytokines.

For example, one pro-inflammatory cytokine, IL-1 $\beta$, only increased slightly after platelet activation, while anti-inflammatory molecules such as IL-4 and IL-10, increased more than five times $[23,24]$. Some in vitro studies confirm that intraarticular PRP injections can influence the entire joint environment [25]. The chemo-attractant activity of PRP may recruit other cells heal damaged tissues [26]. The role of PRP in modulating inflammation which is PRP related with both pro and anti-inflammatory activities is complex. The initial action is pro-inflammatory which is followed by a limitation of the inflammatory response decreasing inflammatory molecules and preventing chemotaxis [27,28]. Regulation of joint inflammation explains how PRP treatment reduces pain favoring patient improvement. PRP injections are safe. No adverse events have been reported in the literature, and there are only few reports of immediate pain and swelling [29,30]. Studies agree on the clinical benefits of PRP injection as compared to saline solution or viscosupplementation injection [24,31,32]. In vitro and preclinical studies show PRP specific chondrogenic growth factors: PDGF, which may stimulate proliferation and collagen synthesis, TGF-beta, which may enhance chondrocyte synthetic activity, matrix production, and cell proliferation and decrease the catabolic activity of IL-1, and FGF which promotes different anabolic pathways. The anti-inflammatory and anti-apoptotic effect of PRP inhibits apoptotic related factors [33-35].

Storage procedures are key. Freeze PRP alters platelet function and lifespan, and negatively change the growth factors release pattern favoring the accumulation of pyrogenic cytokines and increasing the risk of bacterial proliferation. We use fresh PRP and inject it immediately after preparation. This is very important for successful treatment [12]. Several studies have shown the potentially beneficial effect of PRP in promoting cellular anabolism and tissue regeneration. These studies provide the rationale for the application of platelet concentrates in humans $[8,23]$. The 
mechanism of action of PRP is not yet fully understood, several aspects must be studied to optimize the procedure and improve the potential of this biological minimally invasive approach.

\section{Conclusion}

All patients benefitted from PRP therapy. The greatest improvement was observed at 3 months evaluation. At 6 months, results are quite similar, but 12 months after treatment $B E, V A S$, JP, HJHS began to increase. We believe that a second PRP injection could help to protect patient's joint health.

\section{References}

1. Mulder K, Llinás A (2004) The target joint. Haemophilia 10(4): 152-156.

2. Van Dijk K, Fischer K, Van Der Bom JG, Grobbee DE, Van Den Berg HM (2005) Variability in clinical phenotype of severe haemophilia: the role of the first joint bleed. Haemophilia 11(5): 438-443.

3. O'Hara J, Walsh S, Camp C, Mazza G, Carroll L (2018) The impact of severe A haemophilia and the presence of target joints. Health Qual Life Outcomes 16(1): 84.

4. Roosendaal G, Vianen M, Wenting M, Cornelis Van Rinsum (1998) Iron deposits and catabolic Properties of synovial tissue from patients with haemophilia. J Bone Joint Surg 80(3): 540-545.

5. Van Vulpen L, Schutgens R, Coeleveld K, Els C Alsema, Goris Roosendaal, et al. (2015) IL-1b, in contrast to TNFa, is pivotal in blood induced cartilage damage and is a potential target for therapy. Blood 126(19): 2239-2246.

6. Dinarello CA (2011) A clinical perspective of IL-1b as the gatekeeper of inflammation. Eur J Immunol 41(5): 1203-1217.

7. Kanwat H, Singh DM, Kumar CD, Alka B, Biman S, et al. (2018) The effect of intra-articular allogenic platelet rich plasma in Dunkin-Hartley guinea pig model of knee osteoarthritis. Muscles Ligaments Tendons J 7(3): 426-434.

8. Fortier LA, Barker JU, Strauss JE, Mccarell TM, Cole BJ (2011) The role of growth factors in Cartilage. Clin Orthop Relat Res 469(10): 2706-2715.

9. Boswell SG, Cole BJ, Sundman EA, Karas V, Fortier LA (2012) Platelet rich plasma: a milieu of bioactive factors. Arthroscopy 28(3): 429-439.

10. Foster TE, Puskas BL, Mandelbaum BR, Gerhardt MB, Rodeo SA (2009) Platelet-rich plasma: from basic science to clinical applications. Am J Sports Med 37(11): 2259-2272.

11. Kon E, Mandelbaum B, Buda R, Filardo G, Delcogliano M, et al. (2011) Platelet-rich plasma intraarticular injection versus hyaluronicacid viscosupplementation as treatments for cartilage pathology: from early degeneration to osteoarthritis. Arthroscopy 27(11): 1490-1501.

12. Kon E, Filardo G, Di Matteo B , Marcacci M (2013) PRP For the Treatment of Cartilage Pathology. Open Orthop J 7: 120-128.

13. Anitua E, Andia I, Ardanza B, Nurden P, Nurden AT (2004) Autologous platelets as a source of proteins for healing and tissue regeneration. Tromb Haemost 91(1): 4-15.

14. Ornetti, G Nourissat, F Berenbaum, J Sellam, P Richette, et al. (2016) Does platelet- Rich plasma have a role in the treatment of osteoarthritis? J Bone Spine Rev Rhum 83(1): 31-36.

15. Laver L, Marom N, Dnyanesh L, Mei Dan O, Espregueira Mendes J, et al. (2017) PRP for Degenerative Cartilage Disease: A Systematic Review of Clinical Studies. Cartilage 8(4):341-364.

16. Lopez Cabarcos C, Querol F, Moreno S (2009) Recommendations on Rehabilitation in Hemophilia and other congenital coagulopathies. In: Lopez Cabarcos C, Querol F, Moreno S (Eds.), From the Royal Victoria Eugenia Foundation and the Spanish Federation of Hemophilia 13.
17. Caviglia H, Landro ME, Daffunchio C, Galatro G, Douglas Price AL, et al. (2017) Haemophilia. 23(4): 613-619.

18. Schippinger G, Prüller F, Divjak M, Mahla E, Fankhauser F, et al. (2015) Autologous Platelet-Rich Plasma Preparations: Influence of Nonsteroidal Anti-inflammatory Drugs on Platelet Function. Orthop J Sports Med 3(6): 2325967115588896.

19. Lee CH, Cook JL, Mendelson A, Moioli EK, Yao H, et al. (2010) Regeneration of the articular surface of the rabbit synovial joint by cell homing: a proof of concept study. Lancet 376(9739): 440-448.

20. Woodell May J, Matuska A, Oyster M, Welch Z, O’Shaughnessey K, et al. (2011) Autologous protein solution inhibits MMP-13 production by IL1 beta and TNFalpha-stimulated human articular chondrocytes. J Orthop Res 29(9): 1320-1326.

21. Malemud CJ (2010) Anticytokine therapy for osteoarthritis: evidence to date. Drugs Aging 27(2): 95-115.

22. Arend WP, Gabay C (2000) Physiologic. The role of interleukin-1 receptor antagonist. Arthritis Res 2(4): 245-248.

23. Xie X, Zhang C, Tuan R (2014) Biology of platelet-rich plasma and its clinical application in cartilage repair. Arthritis Research \& Therapy 16(1): 204.

24. Bendinelli P, Matteucci E, Dogliotti G, Corsi MM, Banfi G, et al. (2010) Molecular basis of anti-inflammatory action of platelet-rich plasma on human chondrocytes: mechanisms of NF-kappaB inhibition via HGF. J Cell Physiol 225(3): 757-766.

25. Filardo G, Kon E, Roffi A, Di Matteo B, Merli M (2015) Platelet-rich plasma: why intra-articular? A systematic review of preclinical studies and clinical evidence on PRP for joint de-generation. Knee Surg Sports Traumatol Arthrosc 23(9): 2459-2474.

26. Krüger JP, Hondke S, Endres M, Pruss A, Siclari A, et al. (2012) Human platelet-rich plasma Stimulates migration and chondrogenic differentiation of human subchondral progenitor cells. J Orthop Res 30(6): 845-852.

27. Pereira RC, Scaranari M, Benelli R, Strada P, Reis RL, et al. (2013) Dual effect of Platelet lysate on human articular cartilage: a maintenance of chondrogenic potential and a Transient proinflammatory activity followed by an inflammation resolution. Tissue Eng Part A 19 (11-12): 1476-1488.

28. Anitua E, Sánchez M, Nurden AT, Zalduendo MM, De La Fuente M, et al. (2007) Platelet-Released growth factors enhance the secretion of hyaluronic acid and induce hepatocyte growth factor production by synovial fibroblasts from arthritic patients. Rheumatology 46(12): 1769-1772.

29. Filardo G, Kon E, Pereira Ruiz MT, Vaccaro F, Guitaldi R, et al. (2012) Platelet-rich plasma intra-articular injections for cartilage degeneration and osteoarthritis: single- versus double-spinning approach. Knee Surg Sports Traumatol Arthrosc 20(10): 2082-2091.

30. Kon E, Buda R, Filardo G, Di Martino A, Timoncini A, et al. (2010) Platelet-rich plasma: intra-articular knee injections produced favorable results on degenerative cartilage lesions. Knee Surg Sports Traumatol Arthrosc 18 (4): 472-479.

31. Patel S, Dhillon MS, Aggarwal S, Marwaha N, Jain A (2013) Treatment with platelet-rich plasma is more effective than placebo for knee osteoarthritis: a prospective, double-blind, randomized trial. Am J Sports Med 41(2): 356-364.

32. Filardo G, Kon E, Di Martino A, Di Matteo B, Merli ML, et al. (2015) Platelet-rich plasma vs hyaluronic acid to treat knee degenerative pathology: study design and preliminary results of a randomized controlled trial. BMC Musculoskelet Disord 13(1): 229.

33. Marmotti A, Rossi R, Castoldi F, Roveda F, Michielon G, et al. (2015) PRP and Articular Cartilage: A Clinical Update. Biomed Res Int 542502. 
34. Bendinelli P, Matteucci E, Dogliotti G, Corsi MM, Banfi G, et al. (2010) Molecular basis of anti-inflammatory action of Platelet-rich plasma on human chondrocytes: Mechanisms of NF- $\kappa$ B inhibition via HGF. Journal of Cellular Physiology 225(3): 757-766.

\section{ISSN: 2574-1241}

DOI: $10.26717 / B J S T R .2019 .12 .002319$

María Eulalia Landro. Biomed J Sci \& Tech Res

(C) (P) This work is licensed under Creative

Submission Link: https://biomedres.us/submit-manuscript.php
35. Zhu Y, Yuan M, Meng H, Wang AY, Guo QY, et al. (2013) Basic science and clinical application of platelet-rich plasma forcartilage defects andosteoarthritis: a review. Osteoarthritis and Cartilage 21(11): 16271637. 